\title{
Shape Optimization of High-Speed Train with the Speed of $500 \mathrm{kph}$
}

\author{
K. Cui, X.P. Wang, S.C. Hu, T.Y. Gao, and Guowei Yang \\ National Key Laboratory of High temperature Gas Dynamics, \\ Institute of Mechanics, \\ Chinese Academy of Sciences, Beijing 100190, China
}

\begin{abstract}
With the train speed becoming faster and faster, the aerodynamic drag turns to be one of the essential factor that restricts the train speed ascent. However, no public literature, abroad or abroad, has dealt with the flow field and aerodynamic performance of the train with the speed reaching $500 \mathrm{~km}$ per hour. In this paper, an optimization study is carried out to reduce the aerodynamic drag of the high speed train (HST). First of all, a grid-based method is presented to parameterize the head shape of the HST, key variables are obtained by sensitivity analysis. Next, a response surface is constructed based on computational fluid dynamics (CFD) analysis to approximate the relationship of the drag and design variables at 500KPH. Finally, the genetic algorithm is used to optimize the head shape of the HST.
\end{abstract}

Keywords: High speed train, head shape, optimization, RBF, genetic algorithm, computational fluid dynamics.

\section{Introduction}

With the increase in speed of railway trains it became a necessity to consider the aerodynamic effects of airflow over train including the study of induced drag, aerodynamic noise, and wind-related vibrations (Joseph, 2001, Raghunathana, 2002). The current high speed train can achieve a speed of $420 \mathrm{~km}$ per hour (KPH) and this speed can have a significant impact on the aerodynamic drag it generates. Also, due to Reynolds number being more than 20 million in magnitude, the acoustic noise and wind related vibration will become an important issue for design and optimization of the modern trains. The aim is to identify the optimal shape that induces the least drag and minimizes the acoustic noise while still maintaining the structural integrity and other geometric constraints. This type of optimization involves multi-functional constraints which require a complex search algorithm over a multiple design spaces to find a global maximum or minimum. CFD Optimization of a train body can be very expensive and therefore one needs to impose restrictions on the number of functional evaluations that can be performed. Response surface based design optimization helps in reducing the number of real function evaluations necessary to achieve this goal (Alexander, 2006, Keane, 2007). 
With the ascent of the train speed, the experts come to the conclusion that the higher the velocity is, the more intensive the aerodynamics impact exists on the train. As a result, the relative problems on aerodynamics cannot be neglected. Davis found that the aerodynamic drag is proportional to the square of the speed. Because of varies kinds of limitations of constrains, the track width, the train height and so on, the shape of train body hardly changes. Hence, it's better worth designing the reasonable train head for the high speed train to attain the destination of reducing the drag. However, there is little literature available regarding the head shape optimization. This is the motivation of our work.

In this study, a three-dimensional head shape optimization work of a HST is carried out to reduce aerodynamic drag with the speed of 500KPH. A shape increment-based method is brought forward to parameterize the head shape. Initial samples to perform Design of Experiments (DOE) were selected by using both uniform design and Latin Hypercube Sampling technique. CFD computations were employed to calculate the training samples for response surface construction. Genetic algorithm was used as the driver of the optimization.

\section{Initial Head Shape and Parameterization Method}

The real shape of the HST is shown in Figure 1. Due to the optimization is aim at the head shape, the shape is simplified, shown in Figure 2. The head shape is shown in Figure 3.

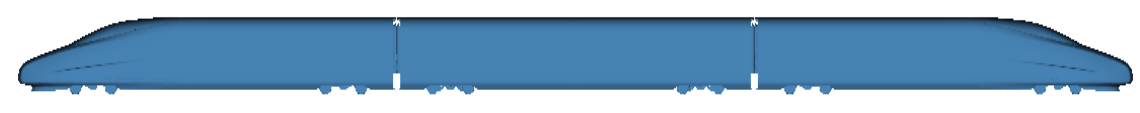

Fig. 1 Real shape of the initial HST

Fig. 2 Simplified shape of the initial HST

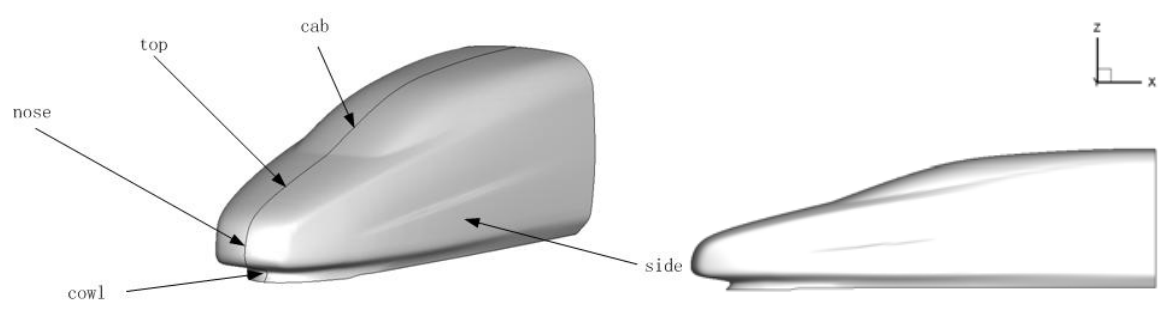

Fig. 3 Head shape of the initial HST 
A shape increment-based method is brought forward to parameterize the head shape. The main idea is as follows. First of all, the head of the HST is divided into several zones, includes the side, the cowl, the nose, the top and the cab, as shown in Figure 3. Next, the head is discretized by structure grids. Based on the parts and the grids above, some key points, includes the peak of the cowl, the peak of the nose, the joint between the top and the cab, and the maximal curvature point of the side. Each point is assigned to control a local zone by given cosine distribution function. Thus the whole head can be controlled by the key points above. Here seven variables is adopted totally, include the $\mathrm{X}$ and $\mathrm{Z}$ coordinate value of the peak point of the cowl, the peak of the nose, the joint between the top and the cab, and the $\mathrm{X}$ coordinate value of the maximal curvature point of the side. Based on the values of the key points and the controlled zones, the increment value of each grid point of the head can be calculated. Finally, by adding the coordinate values of the grid points of the initial shape and the increments, the head shape of the HST can be modified. An example of the head shape modification is shown in Figure 4.

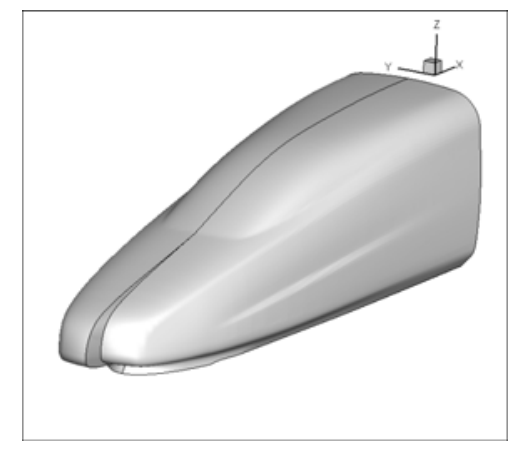

Fig. 4 Example of the head shape modification

\section{Optimization Algorithms}

The genetic algorithm is evolved from the simulation of biological evolution. The optimization mechanism of genetic algorithm is: From randomly generated initial population, adopting a strategy based on survival of fittest to choose the best individual as parents; through the reproduction, crossover and mutation of parent individuals to produce sub-populations. After many generations of evolution, the fitness of the population gradually increased. For a specific optimization problem, the design variables that have the maximum fitness are the optimal solution to the problem at the end of the optimization procedure.

As a global optimization method, genetic algorithm can get the global optimum, but has a large amount of calculation. In this paper, a response surface model was used to instead CFD analysis, so the optimization procedure has a high computational efficiency. And the related settings we used are as follows: 
The population size was 40 , double vector coding was adopted, the number of elite individuals was 2 , the crossover fraction was 0.8 , Gaussian mutation method was applied, with forward migration, and the generations were 50 . The constraint condition was normalized boundary of the design variables, $[-1 ;-1 ;-1 ;-1 ;-1 ;-1 ;-1]$, $[1 ; 1 ; 1 ; 1 ; 1 ; 1 ; 1]$.

Because genetic algorithm is based on principle of probability to search extreme point, so the extreme point will be a little different every time. So for every response surface model, 20 cases were done by genetic algorithm method.

\section{Response Surface Modeling}

The simulation of 3-D flow field around high-speed train by computational fluid dynamics (CFD) is extremely expensive. To optimize the 3-D shape of high-speed train, it requires dozens or even hundreds of CFD analysis. In other words, the cost of calculation and time-consuming is enormous, and sometimes it is even intolerable. In order to efficiently reduce the cost of calculation and timeconsuming, the meta-model was used for the optimization in this paper. Figure 5 showed the procedure of Response Surface method (meta-model) based aerodynamic shape optimization. The meta-model in simple, easy to calculate form, is used to replace the original CFD analysis. Besides, the meta-model also provides an insight to the optimization problem by visualizing the interactions among design variables, objective functions and constrains.

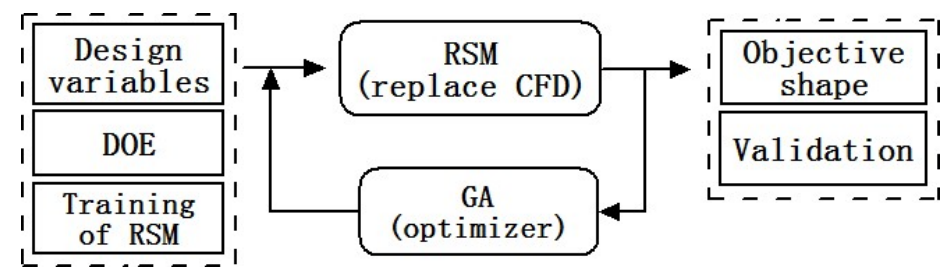

Fig. 5 Procedure of RSM based aerodynamic shape optimization

The establishment of response surface consists of three steeps: 1) Selection of design variables. The $3 \mathrm{D}$ high-speed train nose shape is parameterized with seven design variables. A sensitivity analysis was done before the optimization. As the result showed that all of the design variables are sensitive to the object function (drag coefficient of the high-speed train), all of the seven ones are chosen for the further study. 2) Sampling. Proper Design of Experiments (DOE) is essential for constructing of response surface. Both Orthogonal Arrays and Uniform Design sampling method are used in this study. To refine sample points near the objective zones, an adaptive sampling method is also used in this study. 3) Training and verification of the response surface. Radial Basis Function (RBF) network were choose to construct the response surface. 


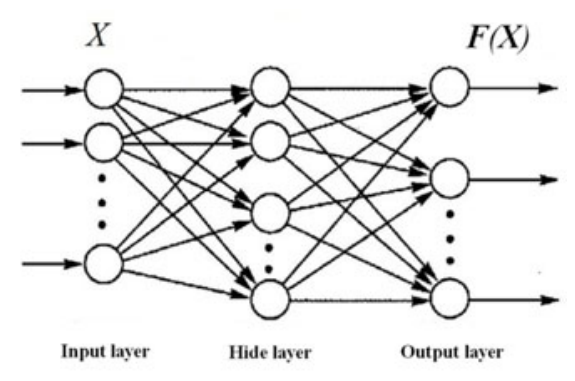

Fig. 6 Radial Basis Function(RBF) net work

The RBF network is shown in Figure 6 In this method, a nonlinear transform from input layer to hidden layer and linear transform from hidden layer to output layer is applied. As the name suggests, the form of these meta-models is a basis function dependent on the Euclidean distance between the sampled data point and point to be predicted. The model can be expressed mathematically as:

$$
F(X)=\sum_{p=1}^{P} w_{p} \varphi(r)=\sum_{p=1}^{P} w_{p} \varphi\left(\left\|X-X^{p}\right\|\right)
$$

Where $w_{p}$ is a real valued weight, $\varphi\left(\left\|X-X^{p}\right\|\right)$ is the basis function, $\left\|X-X^{p}\right\|$ is the Euclidean distance between the points $X$ and $X^{p}, \mathrm{P}$ is the number of sampling points.

\section{Numerical Experiments and Discussions}

\section{(a) Numerical Assumptions}

In this paper, we aim to optimize the streamline part of the train head. Our interest is to minimize the drag coefficient of the target so as to decrease the total drag coefficient of the train, which provides valuable references for the practical design.

In order to decrease the computation time to the affordable condition and to focus our attention to the optimization process, the train has been reasonably simplified, no bogie, no windshield with streamlined surface.

In the optimization process, the used grid is UNSTRUCTURED GRID, the total cell number is 5,000,000, and the scale of the grid on the train wall is $30 \mathrm{~mm}$ and the far field $60000 \mathrm{~mm}$; after the optimization, the validation grid is hybrid grid with the first boundary layer thickness is $0.35 \mathrm{~mm}$ and the total cell number is $5,000,000$. The scale the train far field is $500 \mathrm{~m} \times 300 \mathrm{~m} \times 300 \mathrm{~m}$. 


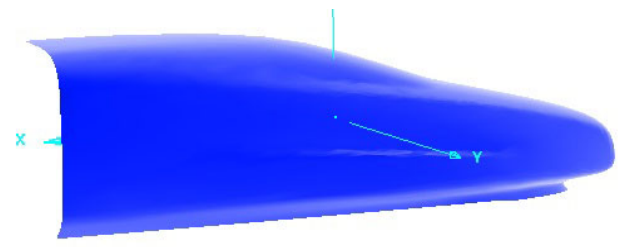

Fig. 7 Original shape

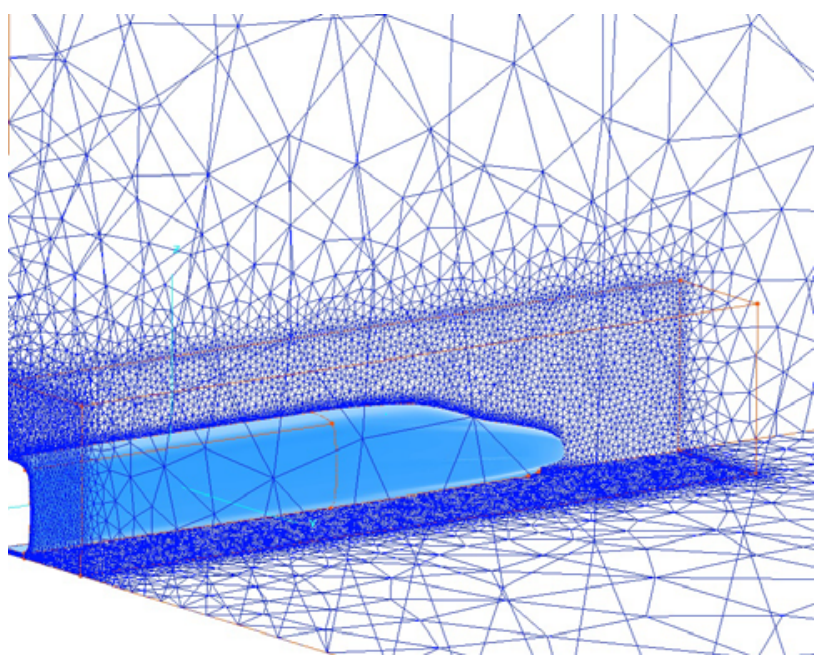

Fig. 8 Schematic of the unstructured grid near the nose shape

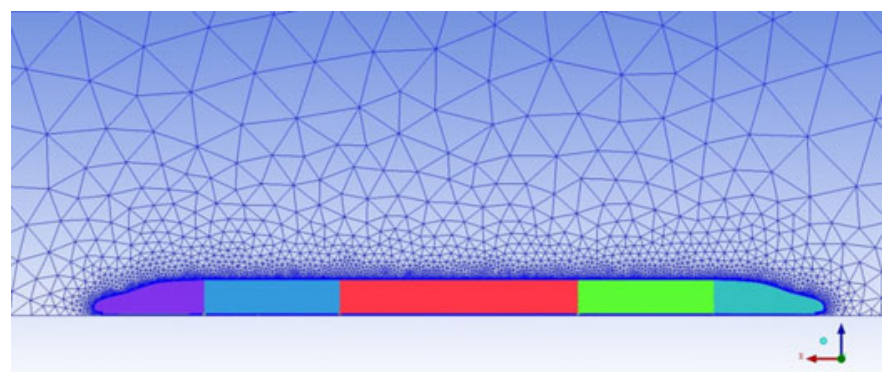

Fig. 9 Schematic of the hybrid mesh around the train

As the velocity of the train is $500 \mathrm{~km} / \mathrm{h}$, we consider the air compressible ideal with the laminar model. The operating pressure is made to 0 . The ground velocity is $138.89 \mathrm{~m} / \mathrm{s}$. far field pressure is the standard atmosphere pressure and the temperature is $288.15 \mathrm{~K}$ and the $\mathrm{Ma}=0.4071$. The outlet pressure is $101325 \mathrm{pa}$ and the total temperature is $297.70 \mathrm{~K}$. 
In the validation process, the computational grid is hybrid mesh and the condition is different from the optimization process in the $\mathrm{k}-\mathcal{E}$ turbulence model.

\section{(b) Procedure And Numerical Results}

Before the optimization, a sensitivity study was done so as to take an analysis if any of the design variables could be ignored. Table 1 showed the results of the sensitivity study. It seems that the first, third and seventh design variables are more sensitive than the others, besides, the other design variables are also sensitive for the objective function (it stands for drag coefficient in this study). As a result, all the seven design variables are chosen for the further study.

Table 1 Results of sensitivity study

\begin{tabular}{ccccc}
\hline CASE & $\mathrm{Cd}$ & $\Delta \mathrm{Cd}$ & $\Delta \mathrm{i}$ & $\Delta \mathrm{Cd} / \Delta \mathrm{i}$ \\
\hline Initial & 0.04360 & --- & --- & --- \\
LMD1 & 0.04334 & -0.00026 & 0.003 & -0.08667 \\
LMD2 & 0.04343 & -0.00017 & 0.010 & -0.01700 \\
LMD3 & 0.04314 & -0.00046 & 0.008 & -0.05750 \\
LMD4 & 0.04320 & -0.00040 & 0.035 & -0.01143 \\
LMD5 & 0.04289 & -0.00071 & 0.025 & -0.02840 \\
LMD6 & 0.04333 & -0.00027 & 0.020 & -0.01350 \\
LMD7 & 0.04310 & -0.00050 & 0.0011 & -0.45455 \\
\hline
\end{tabular}

An adaptive refine RSM is used in the procedure of optimization. The sample points of the RSM are refined twice in this work.

The first RSM is constructed with 26 sampling point, which is designed by uniform design. 20 objective cases of the RSM were found by genetic algorithm method, and 5 of them were chosen to be verified by CFD analysis. The CFD results are shown in Table 2, and it shows the drag coefficient decreased by $19.36 \%$.

Table 2 Optimization results with the first RSM

\begin{tabular}{ccc}
\hline CASE & $\mathrm{Cd}$ & Optimization rate \\
\hline Initial & 0.04360 & \\
Opti1_1 & 0.03519 & $19.29 \%$ \\
Opti1_2 & 0.03516 & $19.36 \%$ \\
Opti1_3 & 0.03610 & $17.2 \%$ \\
Opti1_4 & 0.03563 & $18.28 \%$ \\
Opti1_5 & 0.03785 & $13.19 \%$ \\
\hline
\end{tabular}

11 additional sample points were used for the second RSM, the sample points near the objective zones were refined. Similarly, 20 objective cases of the RSM were found by genetic algorithm method, and 5 of them were chosen to be verified by CFD analysis. The CFD results are shown in Table 3, and it shows the drag coefficient decreased by $21.15 \%$. 
Table 3 Optimization results with the second RSM

\begin{tabular}{ccc}
\hline CASE & Cd & Optimization rate \\
\hline Initial & 0.04360 & \\
Opti2_1 & 0.03490 & $19.95 \%$ \\
Opti2_2 & 0.03438 & $21.15 \%$ \\
Opti2_3 & 0.03486 & $20.05 \%$ \\
Opti2_4 & 0.03468 & $20.46 \%$ \\
\hline
\end{tabular}

For the third RSM, 6 sample points were added to refine the objective zones. The Optimization results are shown in Table 4. It shows the drag coefficient just decrease by $0.8 \%$ when compares to the case Opti2_3, so it is reasonable to believe that twice refined RSM is enough.

Table 4 Optimization results with the third(final) RSM

\begin{tabular}{ccc}
\hline CASE & $\mathrm{Cd}$ & Optimization rate \\
\hline Initial & 0.04360 & \\
Opti3 & 0.03434 & $21.23 \%$ \\
\hline
\end{tabular}

Table 4 shows the final optimization results with $21.23 \%$ decreased of the drag coefficient.

\section{(c) 5.3 Validation}

In this study, we use the hybrid grid to validate the optimization results. from the data showed in Table 5, we can get the conclusion that the validation results is linear to the optimization results, which represents the optimization process successfully.

Table 5 Comparison between the laminar model and turbulence model results

\begin{tabular}{|c|c|c|c|c|c|c|}
\hline CASE & \multicolumn{3}{|c|}{$\begin{array}{c}\text { Laminar model } \\
\text { (Unstructured grid) }\end{array}$} & \multicolumn{3}{|c|}{$\begin{array}{c}\mathrm{k}-\mathcal{E} \text { turbulence model } \\
\text { (Hybrid grid) }\end{array}$} \\
\hline Initial & 0.04315 & 0.00045 & 0.04360 & 0.08131 & 0.02202 & 0.10332 \\
\hline Opti1_2 & 0.03471 & 0.00045 & 0.03516 & 0.05515 & 0.02197 & 0.07713 \\
\hline Opti2_3 & 0.03389 & 0.00048 & 0.03438 & 0.05392 & 0.02343 & 0.07735 \\
\hline Opti3 & 0.03385 & 0.00047 & 0.03434 & 0.05255 & 0.02286 & 0.07541 \\
\hline
\end{tabular}

In order to provide reference value for the practical design, we get two train models with original shape and optimized shape respectively. The Table 6 shows the drag coefficient optimization rate of the streamline part both on the head and the tail part. By optimization, the streamlined part drag coefficient of the head and tail has been decreased by $24.57 \%$ and $28.25 \%$ respectively. 
Table 6 Optimization results of the streamline parts

\begin{tabular}{ccrrrrr}
\hline \multirow{2}{*}{ CASE } & Cd_pressure Cd_viscous & Cd & \multicolumn{2}{c}{ Cd_pressure Cd_viscous } & Cd \\
& \multicolumn{3}{c}{ Streamline part of the head } & \multicolumn{3}{c}{ Streamline part of the tail } \\
\hline Initial & 0.07759 & 0.02209 & 0.09967 & 0.06935 & 0.01831 & 0.08767 \\
Opti3 & 0.05210 & 0.02308 & 0.07518 & 0.04631 & 0.01658 & 0.06290 \\
Optimization rate & & & $24.57 \%$ & & & $28.25 \%$ \\
\hline
\end{tabular}

Table 7 and Table 8 show the optimization results of the train. It proved the optimization of the nose shape is successful and meaningful. Though the streamline part of the train is optimized, the drag coefficient of the whole train also decreased by $19.79 \%$. The pressure contours of the head and the tail are shown in figure 10 and 11 . The pressure distribution in the symmetric plane comparisons between the head and the tail are shown in figure 12 and 13. The difference of the pressure distribution between the initial shape and the optimized one is clearly, especially in the zone around the cowl.

Table 7 Optimization results of different part

\begin{tabular}{cccc}
\hline CASE & Cd_head & Cd_middle & Cd_tail \\
\hline Initial & 0.14062 & 0.05976 & 0.12684 \\
Opti3 & 0.11397 & 0.05340 & 0.09453 \\
Optimization rate & $18.95 \%$ & $10.65 \%$ & $25.48 \%$ \\
\hline
\end{tabular}

Table 8 Optimization results of the train

\begin{tabular}{cccc}
\hline CASE & Cd_pressure & Cd_viscous & $\mathrm{Cd}$ \\
\hline Initial & 0.14954 & 0.17769 & 0.32722 \\
Opti3 & 0.09971 & 0.16217 & 0.26189 \\
Optimization rate & $33.3 \%$ & $8.7 \%$ & $19.97 \%$ \\
\hline
\end{tabular}
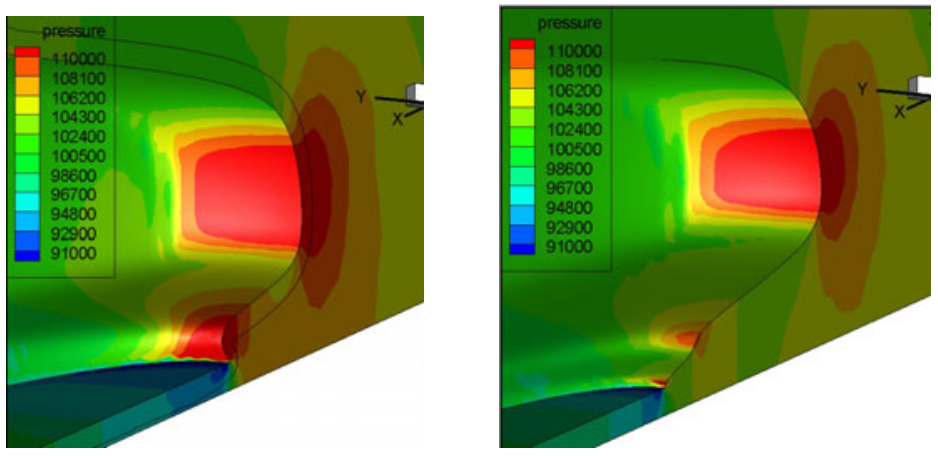

Fig. 10 Pressure contour of the initial (left) and optimized (right) head comparison 

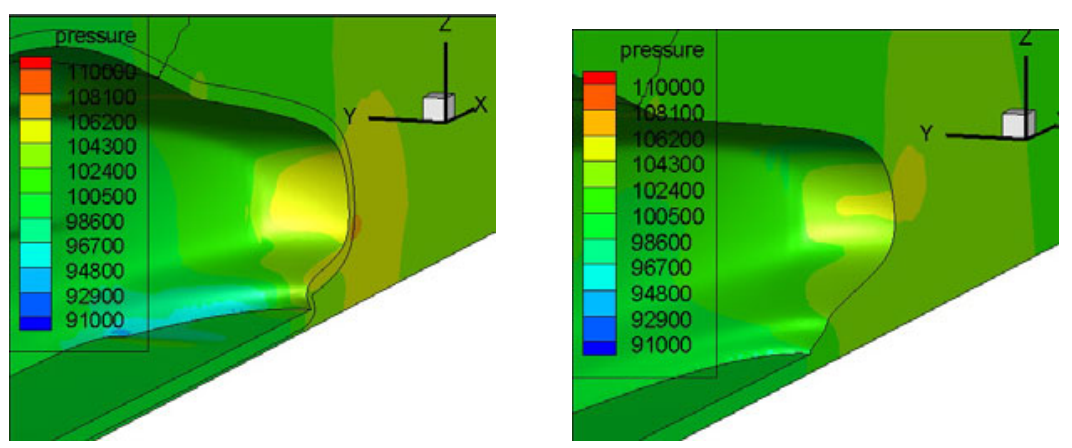

Fig. 11 Pressure contour of the initial (left) and optimized (right) tail comparison

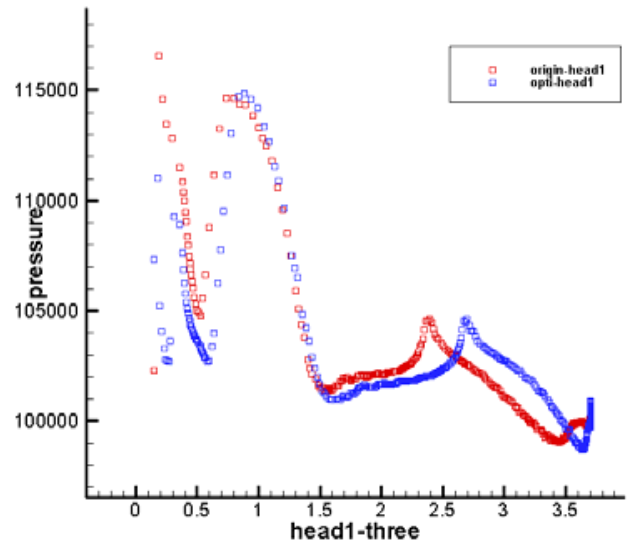

Fig. 12 Pressure distribution of the head part at symmetric plane

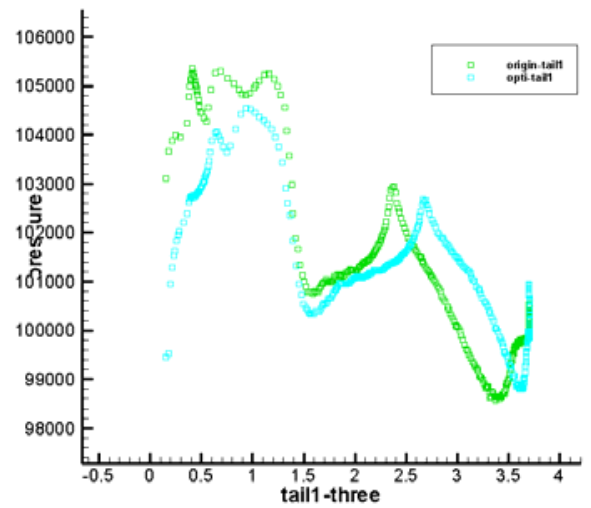

Fig. 13 Pressure distribution of the tail part at symmetric plane 


\section{Conclusions}

An optimization study was conducted to pursue the head shape of HST with lower aerodynamic drag than the original one. The head shape is optimized by adopting the RSM and GA method. The aerodynamic coefficients are evaluated by using simple unstructured grids in the optimization cycle for reducing the computation consumption, while an accurate hybrid grid was used to calculate the aerodynamic performance of the baseline and the optimized shapes for assuring the computational accuracy. It is clear that the drag of the optimized shape is lower than the initial one.

Acknowledgments. The authors gratefully acknowledge the financial support provided by the Natural Science Foundation of China (National Key Project No. 90916013), 973 program under 2011CB711100, and national key technology R\&D program under 2009BAQG12A03.

\section{References}

Alexander, I.J., Forrester, N.W.B., Keane, A.J.: Optimization using surrogate models and partially converged computational fluid dynamics simulations. Phil. Trans. R. Soc. A 462, 2177-2204 (2006)

Joseph, A.S.: Aerodynamics of high-speed trains. Annu. Rev. Fluid Mech. 33, 371-414 (2001)

Keane, A.J., Scanlan, J.P.: Design search and optimization in aerospace engineering. Phil. Trans. R. Soc. A 365, 2501-2529 (2007)

Raghunathana, R.S., Kim, H.D., Setoguchi, T.: Aerodynamics of high-speed railway train. Progress in Aerospace Sciences 38, 469-514 (2002) 